\title{
Le roseau commun (Phragmites australis) : un capital naturel utilisé en litière pour le logement des vaches allaitantes
}

\author{
Daphné Durant, Anne Farruggia, Alexandre Tricheur \\ INRAE, DSLP, F-17450 Saint Laurent de la Prée (France). E-mail : daphne.durant@inrae.fr
}

Reçu le 23 décembre 2020, accepté le 20 septembre 2021, mis en ligne le

Cet article est distribué suivant les termes et les conditions de la licence CC-BY (http://creativecommons.org/licenses/by/4.0/ deed.fr)

Description du sujet. Située en marais, la ferme expérimentale INRAE de Saint Laurent de la Prée cherche des alternatives à la paille de céréales pour le logement de ses vaches allaitantes.

Objectifs. Cette étude vise à explorer l'utilisation du roseau commun (Phragmites australis) en litière pour des vaches allaitantes, à travers ses aspects pratiques mais aussi économiques.

Méthode. Menée deux années consécutives (2018-2019), cette étude a consisté à comparer des litières composées soit de roseau, soit de paille de céréales dans une stabulation libre paillée pour des vaches allaitantes. Les mesures ont porté sur la capacité d'absorption en eau des litières, la propreté des vaches à fréquence et quantité de litière apportée égales, la composition des composts issus de ces litières et enfin, le cout de revient du roseau.

Résultats. La paille a une plus grande capacité d'absorption en eau par rapport au roseau. Les gains de poids moyens de la paille étaient d'environ +63 et $+41 \%$ en années 1 et 2 , respectivement, par rapport au roseau. Cependant, les vaches logées sur du roseau étaient globalement dans un état de propreté semblable à celles hébergées sur de la paille. Le compost de roseau a une valeur fertilisante très proche de celle du compost de paille. Le cout de revient du roseau était de $53 € \cdot \mathrm{t}^{-1} \mathrm{et} 92 € \cdot \mathrm{t}^{-1}$ pour les années 1 et 2 . Il est compétitif par rapport à l'achat de paille (100 €.t-1 de paille livrée) et il l'est d'autant plus que la roselière est proche de la ferme.

Conclusions. Le roseau utilisé comme litière pour le logement des vaches allaitantes pourrait être une alternative à la paille dans les exploitations de marais, à condition d'adopter une gestion des roselières adaptée à leur biologie et à la biodiversité qu'elles abritent.

Mots-clés. Bovin allaitant, capacité d'absorption, litière, ressources naturelles, zones humides.

The common reed (Phragmites australis): a natural capital used as bedding for housed suckler cows

Description of the subject. The INRAE Saint Laurent de la Prée research farm, located in marshland, is looking for alternatives to cereal straw for housing its suckler cows.

Objectives. This study aimed to explore the use of common reed (Phragmites australis) as bedding for suckler cows, through examination of its practical and economic aspects.

Method. Conducted for two successive years (2018-2019), this study consisted of comparing different types of bedding composed of either reed or cereal straw in a free stall for suckler cows. Measurements were taken on the water absorption capacity of the bedding, the cleanliness of the cows with equal frequency and quantity of bedding provided, the composition of the compost from this bedding, and finally, the cost price of the reed.

Results. Straw has a higher water absorption capacity than reed. The average weight gains of straw were approximately +63 and $+41 \%$ in years 1 and 2 , respectively, compared to reed. However, cows housed on reed were generally found to be in a similar state of cleanliness as those housed on straw. Reed compost has a fertilizing value very close to that of straw compost. The cost of reed was $53 € \cdot \mathrm{t}^{-1}$ and $92 € \cdot \mathrm{t}^{-1}$ for years 1 and 2 . The cost of harvesting reed is competitive compared to the purchase of straw (100€ $€ \cdot \mathrm{t}^{-1}$ straw delivered), and it is all the more so as the reed bed is close to the farm.

Conclusions. Reed used as bedding for the housing of suckler cows could be an alternative to straw in marshland farms, provided that reedbeds are managed according to their biology and the biodiversity they contain.

Keywords. Suckler cows, absorption capacity, bedding, natural resources, wetlands. 


\section{INTRODUCTION}

Beaucoup de fermes de polyculture-élevage ne sont pas autonomes en paille pour le logement de leurs animaux. Elles se trouvent dans l'obligation d'en acheter à l'extérieur. Elles sont ainsi soumises aux variations du prix de la paille, mais aussi à des problèmes d'approvisionnement, surtout en période de forte demande (Madelrieux et al., 2017). Comme beaucoup d'exploitations, la ferme de Saint Laurent de la Prée, un dispositif expérimental de l'Institut national de recherche pour l'agriculture, l'alimentation et l'environnement (INRAE), cherche l'autonomie et l'économie en intrants (Durant et al., 2020) mais doit certaines années acheter de la paille directement livrée «dans la cour » et à un

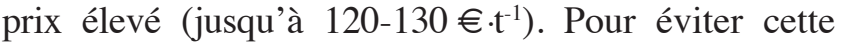
dépendance vis-à-vis de l'extérieur, la ferme explore actuellement d'autres sources de litière. Comme ses activités agricoles doivent être le plus possible en adéquation avec les principes de l'agroécologie (Altieri, 1996; Gliesmann, 1998), dont celui qui consiste à préserver les ressources naturelles présentes localement (en maintenant leur capacité de renouvellement) et les services écosystémiques associés, la question posée est donc: quelles ressources naturelles des marais littoraux atlantiques (contexte dans lequel s'exercent les activités de la ferme) pourraient servir de litière ?

Dans la plupart des élevages bovins, la paille de céréales constitue la première source de litière (Rousset et al., 2014). Mais depuis plusieurs années, des alternatives ont été testées avec succès comme par exemple le Miscanthus, les plaquettes de bois ou le sable dolomitique (Berthelot et al., 2003 ; Capdeville \& Ménard, 2003 ; Van Weyenberg et al., 2015 ; Malterre, 2019). Dans les marais, le roseau commun (Phragmites australis [Cav.] Trin. ex Steud.), constituant dans certaines zones de grandes étendues de roselières, pourrait être testé comme litière. Il s'agit d'une grande graminée vivace de la famille des Poacées largement répandue dans les zones humides du globe (E.P.A., 1985). Depuis des temps très anciens, le roseau est récolté pour divers usages, par exemple pour la couverture végétale des toits des chaumières, comme matériau pour l'industrie, source de bioénergie ou dans les procédés de traitement des eaux (Schricke,1988; Goergen, 1996), dont ceux liés à l'agriculture (Köbbing et al., 2013). En France, il a longtemps été utilisé comme matelas de matières végétales pour le bétail logé en bâtiments et plus marginalement comme fourrage grossier. On l'appelait alors « la litière des marais » ou « la paille des marais » (E.P.A., 1985). Aujourd'hui, il est encore utilisé marginalement par des élevages dans certaines zones humides, comme par exemple dans le Parc Naturel Régional de Brière (Divay et al., 2018). En Charente-Maritime, le département où se situe la ferme expérimentale, le roseau n'est plus exploité. À notre connaissance, seuls deux éleveurs dans le Val de Trézence (secteur du cours d'eau La Boutonne) l'utilisent en le mélangeant à de la paille. Malgré le fait que les surfaces de roselières se soient réduites depuis des dizaines d'années dans ce département (Le Barz et al., 2009 ; Loïc Jomat, com. pers.), l'hypothèse faite ici est que le roseau pourrait retrouver une place dans les exploitations de marais, en particulier celles conduites en agriculture biologique (AB), qui cherchent à mieux valoriser les ressources naturelles du milieu, tout en les préservant.

Constatant l'absence de témoignages ou de connaissances scientifiques dans la littérature quant à l'usage du roseau en élevage (voir cependant Köbbing et al., 2013 ; Divay et al., 2018), une expérimentation consistant à comparer des litières à base de roseau commun ou de paille a été menée deux années consécutives sur la ferme expérimentale. Les objectifs étaient :

- de produire des références scientifiques sur la litière de roseau en comparaison à une litière plus classique à base de paille de céréales (ou d'un mélange « pailleroseau »);

- d'aborder les aspects pratiques et économiques de cet usage ;

- de discuter des possibles valorisations des roselières sur le plan aussi bien environnemental qu'agricole.

\section{MATÉRIEL ET MÉTHODES}

\subsection{Site d'étude et dispositif expérimental}

La ferme expérimentale conduit un système de polyculture-élevage en $\mathrm{AB}$ dans les marais de Rochefort-sur-mer (45 $58^{\prime} 52^{\prime \prime}$ Nord, $0^{\circ} 02^{\prime} 28^{\prime \prime} \mathrm{O}$; Durant \& Kernéiis, 2010 ; Durant et al., 2020). Elle s'étend sur 160 ha et conduit un troupeau de 55-60 vaches allaitantes de race Maraîchine (ainsi que la suite, soit au total 130-140 animaux) qui pâturent les prairies naturelles de début avril à début novembre. Dans cet élevage, le troupeau est hébergé en stabulation libre durant cinq mois de l'année, de début novembre à début avril. Ce mode d'hébergement implique des besoins en paille importants (environ 120-130t par an), jugés incompressibles par les pilotes de la ferme et les animaliers, car indispensables au bien-être des animaux. La paille provient de la culture des céréales sur la ferme et d'achats de paille « derrière la moissonneuse » auprès de fermes voisines.

L'étude a eu lieu dans la stabulation de la ferme expérimentale au cours de trois périodes de 36,39 et 27 jours chacune (essais 1, 2 et 3 ) de novembre 2018 à février 2019 et d'une période de 43 jours (essai 4) d'octobre à décembre 2019 (Tableau 1). Les essais 1 et 4 ont commencé respectivement 6 et 7 jours après 
l'entrée en stabulation des vaches, de façon à ce que celles-ci soient toutes au même stade de propreté dès le début. Les litières ont été testées sur quatre lots d'animaux hébergés dans quatre cases de surfaces sensiblement égales (environ $75 \mathrm{~m}^{2}$ chacune - sans compter une surface de $38 \mathrm{~m}^{2}$ non paillée au niveau des cornadis, Figure 1). Les lots n'étant pas composés des mêmes types d'animaux (vaches avec ou sans leur veau) et ne correspondant donc pas aux mêmes chargements, les cases suivantes ont été comparées deux à deux :

- cases 1 et 3 , avec chacune un lot de 12 vaches sans veau (lot en vêlage de printemps);

- cases 2 et 4, avec chacune un lot de 10 vaches avec leur veau (lot en vêlage d'automne).

Les veaux restaient avec leur mère pour les tétées du matin et du soir, mais séjournaient dans une cabane à veaux (une par lot; surface $=14 \mathrm{~m}^{2}$ ) le reste du temps. Un taureau a été introduit dans chacune de ces deux cases, respectivement les 4 décembre 2018 et 20 novembre 2019 pour les besoins de mise à la reproduction.

Nous avons veillé à respecter la surface minimale par animal du cahier des charges de l'AB, à savoir $7 \mathrm{~m}^{2}$ par vache (poids moyen d'une Maraîchine = $650-700 \mathrm{~kg}$ ) et $8,5 \mathrm{~m}^{2}$ par couple mère-veau. Le poids moyen des vaches à l'entrée en stabulation n'était pas significativement différent entre les cases 1 et 3 (tests de Mann-Whitney : $\mathrm{U}=44, p=0,11$ en 2018 et $\mathrm{U}=$

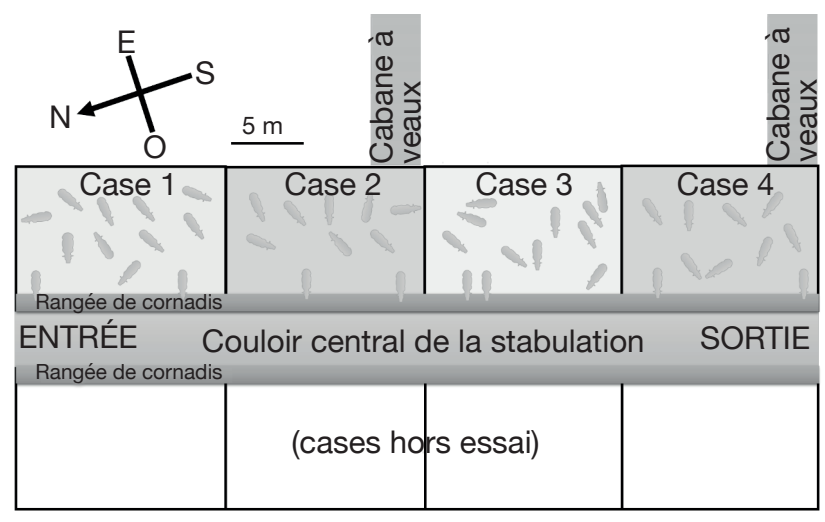

Figure 1. Schéma (vue de dessus) des quatre cases concernées par le dispositif expérimental dans la stabulation - Diagram (top view) of the four boxes concerned by the experimental device in the stall.

$34, p=0,23$ en 2019) ou entre les cases 2 et $4(\mathrm{U}=49$, $p=0,67$ en 2018 et $\mathrm{U}=30, p=0,13$ en 2019).

Les modalités suivantes ont été comparées : « paille » $v s$ « roseau »; « paille » $v s ~ «$ paille + roseau » pour l'année 1 et «paille»vs « roseau », «paille»vs « faux-roseau » pour l'année 2 (Tableau 1). Selon les essais, plusieurs types de paille ont été utilisés. Durant l'automne-hiver 2018-2019, l'essai 1 a été conduit avec de la paille d'orge, assez cassante, ayant deux années de stockage. Les essais 2 et 3 ont été conduits avec de la paille de blé de meilleure qualité, n'ayant qu'une année de stockage. Au cours de l'automne-

Tableau 1. Conditions des essais conduits lors des deux années de l'étude - Conditions of the trials conducted during the two years of the study.

\begin{tabular}{|c|c|c|c|c|}
\hline & \multicolumn{3}{|l|}{ Année 1 } & \multirow{2}{*}{$\begin{array}{l}\text { Année } 2 \\
\text { Essai } 4\end{array}$} \\
\hline & Essai 1 & Essai 2 & Essai 3 & \\
\hline Période & Du $12 / 11$ au $17 / 12 / 2018$ & $\begin{array}{l}\text { Du 21/12/2018 au } \\
28 / 01 / 2019\end{array}$ & $\begin{array}{l}\text { Du 01/02 au } \\
27 / 02 / 2019\end{array}$ & $\begin{array}{l}\text { Du 21/10 au } \\
02 / 12 / 2019\end{array}$ \\
\hline Durée de l'essai & 36 jours & 39 jours & 27 jours & 43 jours \\
\hline Cases $1 \& 3$ & Paille $v s$ roseau & Paille $v s$ roseau & Paille $v s$ roseau & Roseau vs paille \\
\hline Cases $2 \& 4$ & Paille $v s$ roseau + paille & Paille $v s$ roseau + paille & Paille $v s$ roseau + paille & Faux-roseau $v s$ paille \\
\hline \multirow[t]{2}{*}{ Vaches par case } & $12($ cases 1,3$)$ & $12($ cases 1,3$)$ & $12 * *($ cases 1,3$)$ & $12(\text { cases } 1,3)^{* * *}$ \\
\hline & $10 *($ cases 2,4$)$ & $10 *($ cases 2,4$)$ & $10 *($ cases 2,4$)$ & $10($ cases 2,4$)$ \\
\hline $\begin{array}{l}\text { Quantité de } \\
\text { litière par } \\
\text { paillage }\end{array}$ & $\begin{array}{l}1 \text { botte de paille ou } \\
1 / 2 \text { balle de roseau }\end{array}$ & $\begin{array}{l}1 \text { botte de paille ou } \\
1 / 2 \text { balle de roseau }\end{array}$ & $\begin{array}{l}2 \text { bottes de paille ou } \\
1 \text { balle de roseau**** }\end{array}$ & $\begin{array}{l}2 \text { bottes de paille ou } \\
1 \text { balle de roseau }\end{array}$ \\
\hline $\begin{array}{l}\text { Type de paille } \\
\text { utilisée }\end{array}$ & $\begin{array}{l}\text { Paille d'orge } \\
\text { (cassante, } 2 \text { années de } \\
\text { stockage) }\end{array}$ & $\begin{array}{l}\text { Paille de blé } \\
\text { (bonne qualité, } 1 \text { année } \\
\text { de stockage) }\end{array}$ & $\begin{array}{l}\text { Paille de blé } \\
\text { (bonne qualité, } 1 \text { année } \\
\text { de stockage) }\end{array}$ & $\begin{array}{l}\text { Paille de blé } \\
\text { (bonne qualité, paille } \\
\text { de l'année) }\end{array}$ \\
\hline
\end{tabular}

* : la case 4 compte 11 animaux au lieu de 10 - box 4 has 11 animals instead of $10 ; * *$ : une vache retirée de la case 1 dès le début de 1'essai 3 - one cow removed from box 1 at the beginning of trial 3 ; ***: le 15 novembre 2019, 2 vaches ont été retirées de chacune des cases 1 et 3 - on November 15, 2019, 2 cows were removed from each of boxes 1 and 3 ; ****: essai 3, dans $30 \%$ des paillages, cette quantité a quelquefois été divisée par 2 du fait d'une litière peu sale - test 3 , in $30 \%$ of the mulches, this quantity was sometimes divided by 2 because of a little dirty bedding. 
hiver 2019-2020, l'essai 4 a été réalisé avec de la paille de blé (récoltée courant de l'été 2019). Le poids moyen des bottes de paille était de $185 \mathrm{~kg}$ en année 1 , contre $165 \mathrm{~kg}$ en année 2.

Le paillage des cases a été réalisé trois fois par semaine à l'aide d'une pailleuse. Des quantités de litière similaires ont été distribuées dans chaque case. Lors des deux premiers essais, constatant que ces quantités ne permettaient pas de maintenir les animaux suffisamment propres, celles-ci ont été augmentées à partir de l'essai 3 : en moyenne $185 \mathrm{~kg}$ de paille et $192 \mathrm{~kg}$ de roseau par case pour les essais 1 et 2 contre, respectivement, $370 \mathrm{~kg}$ et $385 \mathrm{~kg}$ pour l'essai 3 ou $330 \mathrm{~kg}$ et $375 \mathrm{~kg}$ pour l'essai 4. Plutôt que de changer de qualité de paille, cette solution s'est imposée à nous car nous n'avions pas d'autre paille à notre disposition et parce que nous supposions que cet ajustement aurait été celui d'un éleveur constatant que ses animaux sont sales.

\subsection{La récolte du roseau}

Un préalable à la conduite de l'étude a consisté à se renseigner sur le matériel contemporain le plus adapté pour la fauche et le pressage du roseau. Avec l'avis d'un entrepreneur en travaux agricoles, une faucheuse conditionneuse (faucheuse arrière CLAAS, modèle DISCO 3000 Flapgrouper) a été testée et s'est révélée adéquate. Une roselière d'1,3 ha, située sur la réserve de chasse et de faune sauvage de la cabane de Moins (à $10 \mathrm{~km}$ de la ferme) a été fauchée le 6 septembre 2018. Le roseau ainsi coupé a été mis en andain et laissé à sécher au soleil pendant 7 jours. Le pressage a ensuite eu lieu le 13 septembre 2018 avec une presse à balles rondes KRONE (modèle Comprima CV 150 XC) muni d'un « rotocut », élément indispensable à prévoir sur la machine pour pouvoir conditionner le roseau en brins de $30-40 \mathrm{~cm}$ et ainsi faciliter sa distribution ultérieure à la pailleuse. Au total, 20,4 t de roseau (soit 53 balles d' $1,40 \mathrm{~m}$ de diamètre; poids moyen d'une balle : $385 \mathrm{~kg}$ ) ont été récoltées.

En année 2, une roselière d'1,1 ha située dans le Val de Trézence (à $37 \mathrm{~km}$ de la ferme) a été fauchée le 13 septembre 2019. Elle était moins uniforme en termes de végétation que la précédente, avec une partie de sa surface composée de roseau commun (Phragmites australis) mais aussi de grand carex (Carex sp.) et de baldingère faux-roseau (Phalaris arundinacea L.). Une autre faucheuse a été utilisée (faucheuse frontale KRONE, modèle EasyCut, sans conditionneuse) qui s'est avérée un peu moins performante. Le mode de séchage a été le même que l'année précédente, les fortes chaleurs de l'été 2019 ayant cependant permis de ramasser le roseau seulement 6 jours après la fauche. Au total, 16,1 t (soit 43 balles d'1,40 m de diamètre ; poids moyen d'une balle : $375 \mathrm{~kg}$ ) ont été récoltées.
$\mathrm{Au}$ moment du pressage, les balles composées de roseau commun ont été distinguées des autres balles de «faux-roseau » (nombre de balles des deux types approximativement égal).

Le niveau de production des roselières a été estimé à partir de la biomasse extraite de la coupe. Il était de 15,7 et $14,6 \mathrm{t} \cdot \mathrm{ha}^{-1}$, en années 1 et 2 respectivement. En dehors des coupes réalisées pour les besoins de l'étude, les modalités de gestion des deux roselières étaient les suivantes : un tiers de la surface de la roselière de la cabane de Moins est coupé chaque année et la coupe est suivie d'une opération de brûlage, alors que la roselière du Val de Trézence est généralement broyée tous les 2-3 ans. Le résultat de ce broyage reste sur place, il n'est pas exporté. Cette accumulation de litière peut cependant à terme entrainer un atterrissement de la roselière.

\subsection{Les mesures et observations}

Évaluation de la capacité d'absorption en eau des litières. Afin de déterminer le pouvoir absorbant des différents types de litières, le protocole décrit par Gasser (2007) a été reproduit en laboratoire. Des échantillons d'environ $200 \mathrm{~g}$ de litière ont été placés dans des casiers métalliques $(30 \mathrm{~cm}$ de long $\times 20 \mathrm{~cm}$ de large $\times 10 \mathrm{~cm}$ de haut), eux-mêmes emballés dans un tissu moustiquaire pour y retenir les fragments de litière. Les casiers ont été immergés dans un grand bac d'eau selon des durées croissantes : 3, 6, 22, 27, 44, 66 et $138 \mathrm{~h}$. Après chaque immersion, les casiers ont été égouttés $1 \mathrm{~h}$, puis pesés. Ils ont ensuite été replongés dans l'eau en attente de la pesée suivante. De l'eau a quelque fois été ajoutée dans le bac de façon à ce que les casiers soient maintenus totalement immergés.

En année 1, la capacité d'absorption en eau de la paille d'orge (essai 1) a été comparée à celle de roseau et d'un mélange «paille + roseau » (Tableau 2). Cette même manipulation a été refaite avec la paille de blé des essais 2 et 3 . Trois répétitions par type de litière ont été réalisées à chaque manipulation, soit 9 échantillons au total. En année 2, les casiers ont été remplis de paille de blé, de roseau et de faux-roseau de l'essai 4. Trois répétitions par type de litière ont également été réalisées. Les valeurs des trois répétitions ont été moyennées pour ne donner qu'une seule valeur par temps d'immersion.

\subsection{Composition chimique du matériel végétal}

La paille et le roseau ont fait l'objet d'analyses pour évaluer en particulier leur contenu en fibres (NDF et ADF - Neutral/Acid Detergent Fiber) selon la méthode de Van Soest et al. (1991) et en cendres (résidus après l'incinération complète des matières organiques). Un échantillon de chaque type de litière a été prélevé lors 
Tableau 2. Composition des casiers lors des deux années de l'étude - Composition of the racks during the two years of the study.

\begin{tabular}{|c|c|c|c|c|c|c|}
\hline & \multicolumn{3}{|c|}{ Année 1 (2018-2019) } & \multicolumn{3}{|c|}{ Année 2 (2019-2020) } \\
\hline $\begin{array}{l}\text { Composition } \\
\text { des casiers }\end{array}$ & $\begin{array}{l}\text { Paille d'orge } \\
\text { (ou de blé) } \\
\text { (3 répétitions) }\end{array}$ & $\begin{array}{l}\text { Roseau } \\
\text { (3 répétitions) }\end{array}$ & $\begin{array}{l}\text { Paille }+ \text { roseau } \\
\text { (3 répétitions) }\end{array}$ & $\begin{array}{l}\text { Paille de blé } \\
\text { ( } 3 \text { répétitions) }\end{array}$ & $\begin{array}{l}\text { Roseau } \\
\text { (3 répétitions) }\end{array}$ & $\begin{array}{l}\text { Faux-roseau } \\
\text { ( } 3 \text { répétitions) }\end{array}$ \\
\hline $\begin{array}{l}\text { Poids de litière } \\
\text { (sec) }\end{array}$ & $200 \mathrm{~g}$ & $200 \mathrm{~g}$ & $100 \mathrm{~g}+100 \mathrm{~g}$ & $200 \mathrm{~g}$ & $200 \mathrm{~g}$ & $200 \mathrm{~g}$ \\
\hline $\begin{array}{l}\text { Dates de } \\
\text { réalisation }\end{array}$ & \multicolumn{3}{|c|}{$\begin{array}{l}\text { Du } 6 \text { au } 12 \text { novembre } 2018 \text { (paille d'orge) } \\
\text { Du } 19 \text { au } 25 \text { février } 2019 \text { (paille de blé) }\end{array}$} & \multicolumn{3}{|c|}{ Du 19 au 25 novembre 2019} \\
\hline
\end{tabular}

d'un paillage, mis à l'étuve pendant $72 \mathrm{~h}$ à $60^{\circ} \mathrm{C}$, puis envoyé pour analyses au laboratoire INRAE de Lusignan.

\subsection{Observations sur les animaux et les litières en stabulation}

Des notations de propreté des animaux ont été faites deux fois par semaine, le matin vers 8 h 30-9 h (alors que les vaches s'alimentaient à l'auge), à l'aide d'une grille d'observation (adaptée de Bastien et al., 2006). Cette grille est composée de sept niveaux de saleté croissants : «propre », " peu sale - », « peu sale », «peu sale + » ou « sale - » (notations équivalentes), « sale », «sale + » et «très sale ». Dans le même temps, deux paramètres des litières ont été relevés avant le paillage :

- leur température, prise à mi-hauteur de la couche de litière (soit $5-10 \mathrm{~cm}$ de profondeur) grâce à une sonde-thermomètre (Marque Testo, modèle 104-IR). Dans chaque case, six relevés répartis spatialement ont été effectués et les six valeurs moyennées pour obtenir une température moyenne par case ;

- leur état de salissement, estimé en relevant le pourcentage de surface paillée de la case avec une litière encore propre (la litière était considérée comme souillée à partir du moment où elle perdait sa couleur jaune pour devenir marron, salie par les excréments des animaux).

Enfin, toujours lors des matinées des notations, les paramètres d'ambiance du bâtiment (température, hygrométrie) ont été relevés à l'aide de deux capteurs (Marque : TFA, modèle Klimalogg pro) situés à environ $3 \mathrm{~m}$ de hauteur (le premier placé entre les cases 1 et 2 et le second entre les cases 3 et 4 ).

\subsection{Composition des composts issus de ces litières}

Entre deux essais, le curage des cases en expérimentation a eu lieu et les fumiers issus de ces cases ont été stockés dans la fumière couverte de la ferme. Les fumiers de paille et de roseau ont été séparés l'un de l'autre, afin de permettre des prélèvements ultérieurs distincts. Le fumier de la case « roseau + paille » a cependant été mélangé à celui de roseau. Fin mai 2019, les fumiers ont été transportés au champ pour y être compostés en maintenant séparés les deux types de fumier. Ce compostage a été réalisé grâce à deux opérations de retournement d'andains mi-juin et début juillet (22 jours d'intervalle). La température des composts a été relevée tous les 1-2 jour(s) grâce à une sonde (Marque Dramiński Hmm), du 30 mai au 18 juillet 2019. La mesure a été faite à environ $40 \mathrm{~cm}$ de profondeur, à trois endroits de l'andain (dans sa partie haute, moyenne et basse). Enfin, le 25 juillet 2019, trois échantillons de chaque type de compost ont été prélevés avant l'épandage au champ. Les échantillons ont ensuite fait l'objet des analyses suivantes par le laboratoire Auréa AgroSciences de La Rochelle : \% d'humidité, \% de matière organique, $\%$ de matières minérales, teneur en azote total, phosphore et potassium et enfin rapport $\mathrm{C} / \mathrm{N}$ (rapport carbone total/azote total).

\subsection{Calcul du cout de revient du roseau}

Le cout des opérations de récolte a été calculé sur la base des factures fournies par l'entreprise de travaux agricoles à laquelle nous avons fait appel durant les deux années. Les frais de transport des balles de roseau de la première année ont été estimés sur la base de la consommation de carburant pour les trois allers-retours (itinéraire de $10 \mathrm{~km}$ entre la roselière et la ferme), sans prendre en compte cependant le cout de "maind'œuvre » lié à cette charge. Le prix de référence de l'achat de paille a été fixé à $100 € \cdot \mathrm{t}^{-1}$ quand il s'agissait de paille « livrée dans la cour» (selon les prix moyens indiqués dans la presse agricole locale) ou à $60 € \cdot \mathrm{t}^{-1}$ pour de la paille achetée «derrière la moissonneuse » (soit un cout de $35 € \cdot \mathrm{t}^{-1}$ pour la paille + les charges de mécanisation).

\subsection{Analyses des données}

La majorité des analyses a consisté en des comparaisons de moyennes (analyses non paramétriques quand la 
taille des échantillons était < 30). Des tests de MannWhitney (comparaison 2 à 2) ont été réalisés pour vérifier que le poids moyen des vaches (au début des essais 1 et 4) n'était pas significativement différent et pour déterminer si un type de litière absorbait plus d'eau qu'un autre (en ne prenant en compte que les valeurs au niveau du plateau d'absorption). Pour les essais en stabulation, des tests de Kruskal-Wallis ont permis de tester d'éventuelles différences dans la température et l'état général des litières. La chronologie de l'état de propreté des animaux a fait l'objet d'analyses de variance (ANOVA) pour mesures répétées, étant donné la non-indépendance des observations les unes par rapport aux autres (l'état de propreté d'un animal à un temps $t$ étant dépendant de celui au temps $t-1$ ). Il a ainsi été examiné si l'état général extérieur des animaux était différent d'une case à l'autre. Pour cela, une note a été attribuée à chaque catégorie de propreté (de la note 7 pour la catégorie "propre» à la note 1 pour «très sale »). En fonction du pourcentage d'animaux dans chacune des sept catégories, une note globale de propreté par lot d'animaux et par date d'observation a été obtenue. Enfin, les contenus en minéraux et la valeur fertilisante des composts ont fait l'objet de tests de Mann-Whitney, alors que le suivi de leur température a fait l'objet d'une ANOVA à mesures répétées. Les analyses ont été faites avec les logiciels Statistica (version 7.1) et R (version 3.6.1) au seuil de significativité de $5 \%$.

\section{RÉSULTATS}

\subsection{Capacité d'absorption en eau des litières}

En année 1, les résultats des essais 1 et 2 (avec de la paille d'orge et de blé) ont été poolés. La saturation

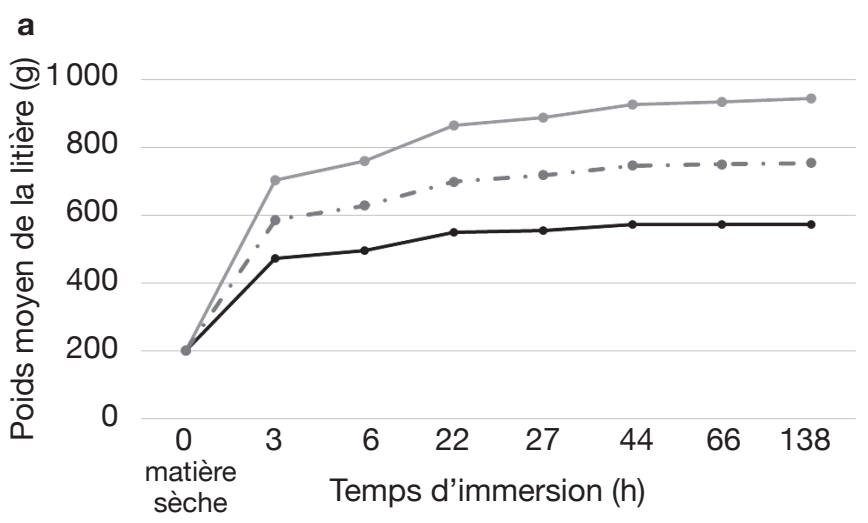

$$
\multimap \text { Paille } \rightarrow \text { Roseau } \leadsto \text { Paille + roseau }
$$

en eau des litières est apparue à partir de la $44^{\mathrm{e}} \mathrm{h}$ d'immersion (Figure 2a ; les écarts-types, trop faibles pour être visibles sur la figure, n'ont pas été représentés - variation de 0,4 à $18,9 \mathrm{~g}$ ), moment où un plateau de saturation apparait. Au niveau de ce plateau, la paille a absorbé significativement plus d'eau (le gain de poids moyen des litières est de $63,5 \%$ ) que le roseau (test de Mann-Whitney : $\mathrm{U}=$ $5,12, p \leq 0,001$ ), mais également plus d'eau (gain de poids de 24,6\%) que le mélange «paille + roseau » $(\mathrm{U}=5,12, p \leq 0,001)$. Le gain de poids sur le mélange est lui aussi plus élevé $(+31,2 \%)$ que pour le roseau seul $(\mathrm{U}=5,12, p \leq 0,001)$. Les analyses de deux constituants de la matière végétale n'ont révélé aucune différence majeure de ces deux types de litière : pour le contenu en fibres (NDF) : 80,5\% pour la paille $v s 77,5 \%$ pour le roseau (pour la paille, moyenne des valeurs pour la paille d'orge et la paille de blé) et pour le contenu en cendres $(6,5 \%$ pour la paille $v s 7,1 \%$ pour le roseau).

En année 2, la saturation des litières a été plus tardive puisqu'elle n'est apparue que vers la $66^{\mathrm{e}} \mathrm{h}$ d'immersion (Figure 2b). Le roseau, quel que soit son type (roseau ou faux-roseau) a absorbé autant d'eau qu'en année 1 (atteinte d'un plateau vers $600 \mathrm{~g}$ pour les deux années). Par contre, la paille de blé utilisée en année 2 était légèrement moins absorbante que celle de l'année 1 (plateau atteint vers $840 \mathrm{~g}$ au lieu de $935 \mathrm{~g}$ environ en année $1 ; \mathrm{U}=2,88, p \leq 0,01$ ). Le gain de poids de la paille était de $+40,9 \%$ par rapport au roseau $(\mathrm{U}=2,88, p \leq 0,01)$ et $+52,2 \%$ par rapport au faux-roseau ( $\mathrm{U}=2,88, p \leq 0,01)$. Les niveaux d'absorption du roseau et du faux-roseau étaient très proches, mais eux aussi significativement différents $(\mathrm{U}=2,88, p \leq 0,01$; le roseau étant $8 \%$ plus absorbant que le faux-roseau).

\section{b}

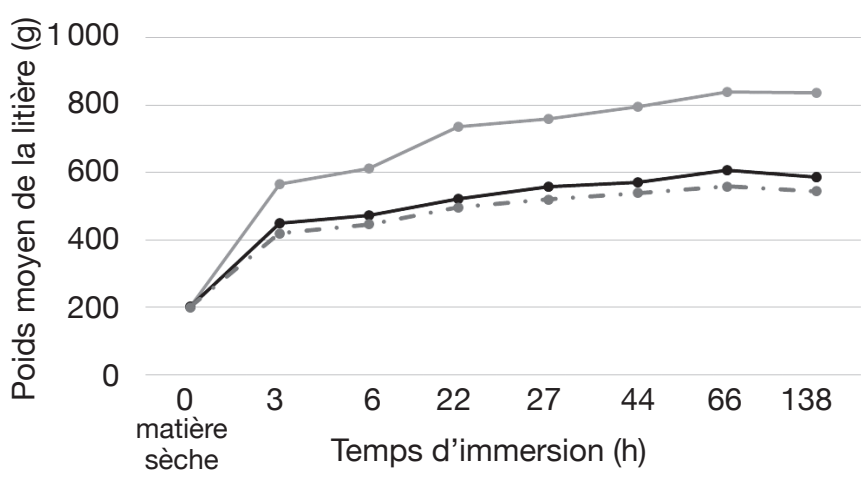

$\multimap$ Paille $\rightarrow$ Roseau $\leadsto$ Faux-roseau

Figure 2. Évolution du poids moyen des litières dans les casiers en fonction des temps d'immersion croissants en année 1 (a) et année 2 (b) - Evolution of the average weight of beddings in the racks as a function of increasing immersion times in year 1 (a) and year $2(\boldsymbol{b})$. 


\subsection{Test du roseau comme litière de substitution pour les vaches allaitantes}

Résultats de l'année 1 : essais 1 à 3. Les trois essais successifs se sont déroulés dans la stabulation où la température ambiante relevée le matin était en moyenne de 8 à $10^{\circ} \mathrm{C}$ et l'hygrométrie de 85 à $90 \%$. De fortes variations de températures ont cependant été observées au cours des essais (amplitude [mini ; maxi] : $1,7^{\circ} \mathrm{C} ; 15,4^{\circ} \mathrm{C}$ ). Le taux d'humidité de l'air a montré de moins grandes variations (amplitude [mini ; maxi] : $78 \% ; 94 \%)$.

La température des litières lors des essais 1 et 2 a varié de 20 à $26^{\circ} \mathrm{C}$ (Tableau 3 ; peu de différences d'une case à l'autre, exception faite de la température dans la case 2 qui était légèrement et significativement supérieure à celle de la case 1). Au cours de l'essai 3, un gradient de température se dégage entre les litières, significatif et décroissant, allant d'environ 34 à $25^{\circ} \mathrm{C}$ des cases 1 à 4 . La case 1 étant la première exposée aux rayons du soleil le matin, il est très probable que cela soit le fait de l'emplacement des cases dans la stabulation. Cette différence de température n'arrivant que lors de l'essai 3, elle serait due :

- au changement de position du soleil dans le ciel au fur et à mesure que la fin de l'hiver approchait ;

- au double de quantité de litière distribué lors de l'essai 3.

Pour les essais 1 et 2, les animaux logés sur la litière de roseau montrent des proportions élevées dans les catégories «peu sale - » et «peu sale », alors que pour la paille, cette répartition est clairement décalée vers les catégories les plus sales («peu sale », « sale », voire «très sale »; Figure 3a). Graphiquement, l'avantage est donc aux vaches logées sur le roseau. Le même type de répartition est constaté pour l'essai 3 (graphe non fourni). L'analyse de ces données, une fois traduites en une note globale de propreté des lots d'animaux, confirme que les vaches sur la litière de roseau avaient une note significativement plus élevée d'1,3 point (lors des essais 1 et $2 ; \mathrm{F}=250,3, \mathrm{dl}=1, p \leq 0,001$; Figure 3b) et de 0,7 point (lors de l'essai $3 ; \mathrm{F}=18,56$, $\mathrm{dl}=1, p \leq 0,05)$ par rapport à celles logées sur de la paille. L'état de la litière n'a cependant montré aucune différence significative d'une case à l'autre, avec un maximum de $10-15 \%$ (essais 1 et $2 ; \mathrm{U}=209, p=$ 0,31 ) et $30-35 \%$ (essai $3 ; \mathrm{U}=38, p=0,82$ ) de la litière qui est restée propre.

Lors des essais 1 et 2 , les vaches logées sur la paille avaient une note moyenne significativement plus élevée de 0,7 point par rapport à celles de la case «paille + roseau $\gg(\mathrm{F}=58,82, \mathrm{dl}=1, p \leq 0,001)$. Lors de l'essai 3 au contraire, aucune litière n'a eu d'avantage sur l'autre $(\mathrm{F}=0,006, \mathrm{dl}=1, p=0,94)$. L'état de la litière n'a montré aucune différence significative d'une case à l'autre (essais 1 et $2: U=177 ; p=0,14$ et essai 3 : $\mathrm{U}=38,5 ; p=0,85)$.

Résultats de l'année 2: essai 4. La température moyenne de l'air ambiant sous la stabulation était de $11^{\circ} \mathrm{C}$ et $l^{\prime}$ hygrométrie de $89 \%$. Les amplitudes [mini ; maxi] de ces deux paramètres étaient : $4,8^{\circ} \mathrm{C}$; $17,6^{\circ} \mathrm{C}$ et $85 \% ; 92 \%$. La case 1 , paillée avec du roseau, ainsi que la case 3 , avec de la paille, a montré une température moyenne significativement supérieure à celle du faux-roseau $(\mathrm{H}=17,73, p \leq 0,0001$; Tableau 3). Les températures des litières de l'année 2 (essai 4) étaient supérieures d'environ $13^{\circ} \mathrm{C}$ à celles de l'année $1(\mathrm{~F}=212,6, p \leq 0,001)$. Ce résultat est à mettre en relation avec la plus grande quantité de litière (le double) distribuée pour maintenir les animaux propres la seconde année.

De manière générale, les animaux ont été maintenus plus propres qu'en année 1 (comparaison des figures 3 et 4). Le niveau de propreté des animaux était identique entre les cases « roseau » et « paille» $(\mathrm{F}=1,21, \mathrm{dl}=1$,

Tableau 3. Température moyenne $\left({ }^{\circ} \mathrm{C} \pm\right.$ écart-type) des litières relevées dans les différentes cases au cours des quatre essais (en années 1 et 2) - Average temperature $\left({ }^{\circ} \mathrm{C} \pm\right.$ standard deviation) of the beddings collected in the different boxes during the four trials (in years 1 and 2 ).

\begin{tabular}{|c|c|c|c|c|c|c|c|}
\hline Année & $\mathrm{N}^{\circ}$ de l'essai & Case 1 & Case 2 & Case 3 & Case 4 & $\begin{array}{l}\text { Nombre de } \\
\text { mesures par } \\
\text { case }\end{array}$ & Test de Kruskal-Wallis \\
\hline & & Paille & Paille & Roseau & $\begin{array}{l}\text { Roseau + } \\
\text { paille }\end{array}$ & & \\
\hline \multirow[t]{4}{*}{ Année 1} & 1 & $22,8 \pm 5,4$ & $25,8 \pm 3,8$ & $23,0 \pm 4,2$ & $23,0 \pm 2,7$ & 9 & $\mathrm{H}=8,44, p=0,037$ \\
\hline & 2 & $22,7 \pm 6,9$ & $21,9 \pm 5,2$ & $19,7 \pm 4,4$ & $19,6 \pm 4,9$ & 11 & $\mathrm{H}=7,52, p=0,056$ \\
\hline & 3 & $33,6 \pm 8,9$ & $29,8 \pm 5,6$ & $26,6 \pm 6,4$ & $25,0 \pm 5,3$ & 9 & $\mathrm{H}=11,27, p=0,01$ \\
\hline & & Roseau & $\begin{array}{l}\text { Faux- } \\
\text { roseau }\end{array}$ & Paille & Paille & & \\
\hline Année 2 & 4 & $41,0 \pm 5,8$ & $32,5 \pm 5,9$ & $38,8 \pm 7,6$ & $37,0 \pm 8,3$ & 12 & $\mathrm{H}=17,73, p=0,0001$ \\
\hline
\end{tabular}


a

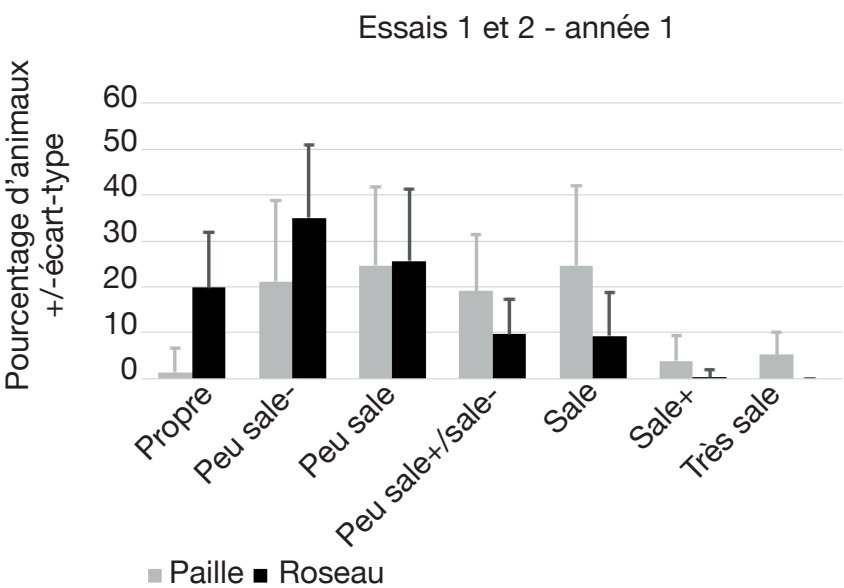

b

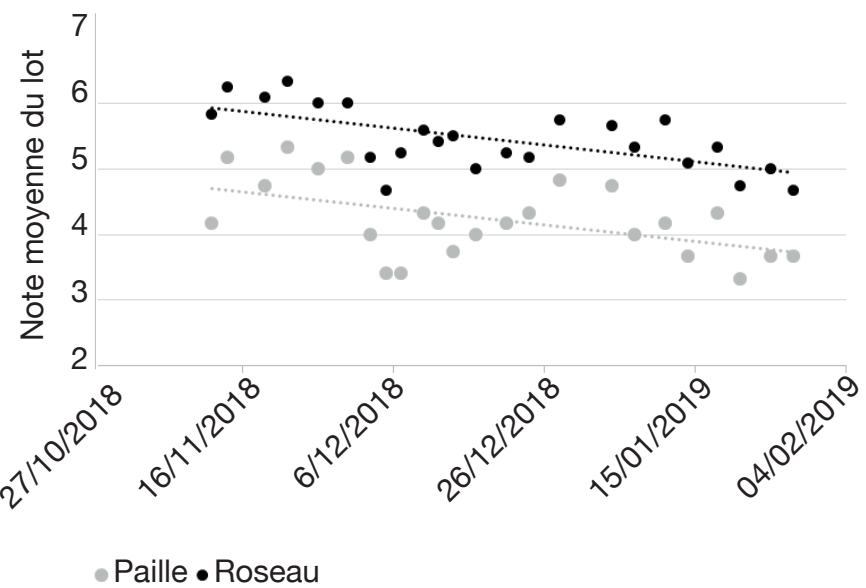

Figure 3. Comparaison de l'état de propreté des vaches dans les cases «paille» $v s$ « roseau » au cours des essais 1 et 2 (moyenne des deux) (a) et évolution des notes moyennes des lots de vaches au cours du temps (b) - Comparison of the cleanliness of cows in straw vs reed boxes during trials 1 and 2 (average of both) (a) and evolution of the average scores of the cow groups over time $(\boldsymbol{b})$.
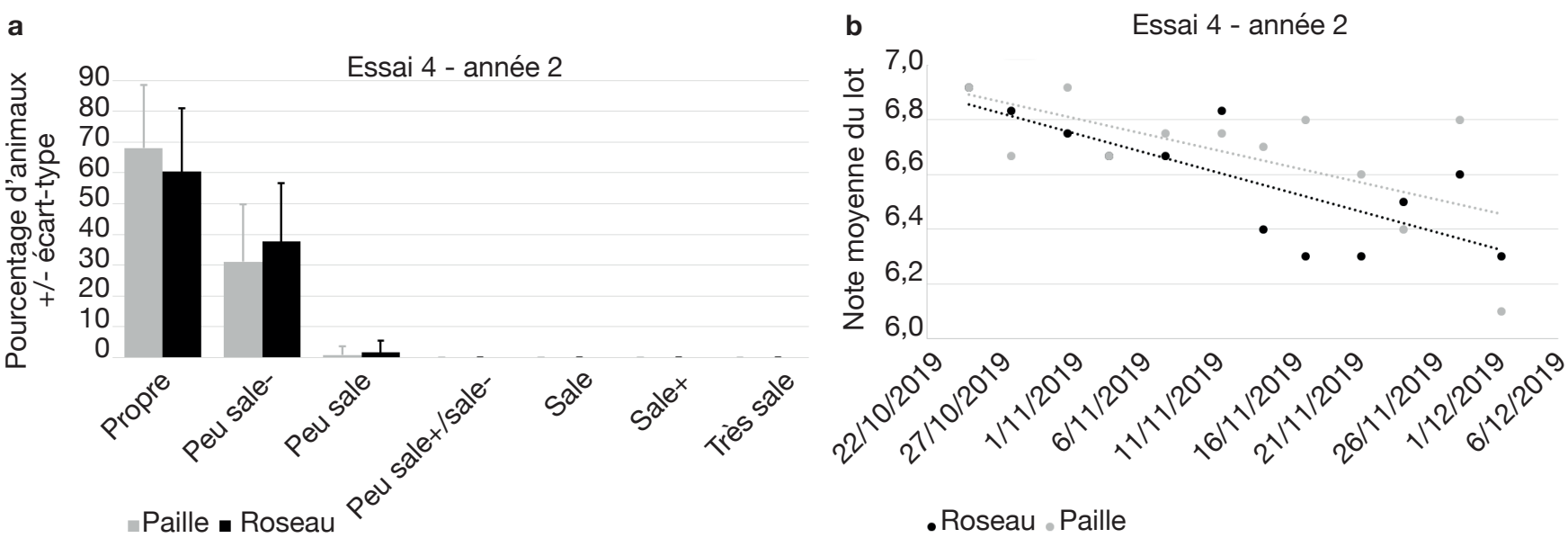

Figure 4. Comparaison de l'état de propreté des vaches dans les cases «paille $v s$ « roseau » au cours de l'essai 4 (a) et évolution des notes moyennes des lots de vaches au cours du temps (b) - Comparison of the cleanliness of cows in straw vs reed boxes during trial $4(\boldsymbol{a})$ and evolution of average scores of cow groups over time $(\boldsymbol{b})$.

$p=0,31 ;$ Figures $\mathbf{4 a}$ et $\mathbf{4 b})$. Ce résultat est également valable entre les cases « paille » et « faux-roseau » $(\mathrm{F}=$ $1,95, \mathrm{dl}=1, p=0,21)$, d'autant que l'état général des litières était lui aussi non significativement différent (paille $v s$ roseau : $\mathrm{U}=25,5, p=0,06$ et paille $v s$ fauxroseau : $\mathrm{U}=50,5, p=0,51$ ).

\subsection{Composition en éléments minéraux et valeur fertilisante des composts}

Il n'y a aucune différence significative dans le contenu en matière minérale entre les deux composts (Tableau 4). Seules les valeurs de phosphore et de potassium se sont révélées légèrement et significativement inférieures pour le compost de roseau $\left(15,9\right.$ et $75,2 \mathrm{~g} \cdot \mathrm{kg}^{-1}$ poids sec, respectivement) comparé au compost de paille $(17,1$ et $87,0 \mathrm{~g} \cdot \mathrm{kg}^{-1}$ poids sec). Les rapports $\mathrm{C} / \mathrm{N}$ (environ 11 ) ne sont pas différents.

Les températures relevées au champ pendant les mois de juin-juillet 2019 au sein des deux types de compost ont varié de $28^{\circ} \mathrm{C}$ à $71^{\circ} \mathrm{C}$. La montée en température du compost de roseau a été significativement plus élevée de $5{ }^{\circ} \mathrm{C}(\mathrm{F}=11,15$; dl $=$ $1 ; p \leq 0,01)$ par rapport à celle du compost de paille.

\subsection{Cout de revient du roseau}

Le cout de revient du roseau était de $53 € \cdot \mathrm{t}^{-1}$ et $92 € \cdot \mathrm{t}^{-1}$, respectivement pour les années 1 et 2 (Tableau 5). Cette différence est due à : 
Tableau 4. Composition en éléments minéraux des composts de paille et de roseau (trois échantillons de chaque) — Mineral composition of straw and reed composts (three samples of each).

\begin{tabular}{llll}
\hline & Compost de paille $(\mathrm{n}=3)$ & Compost de roseau $(\mathrm{n}=3)$ & Test de Mann-Whitney \\
\hline Humidité $(\%$ du produit brut) & $58,2 \pm 8,4$ & $52,1 \pm 2,0$ & $\mathrm{U}=2,0 ; \mathrm{NS}$ \\
Matière organique (\% du produit brut) & $26,3 \pm 5,5$ & $30,5 \pm 1,5$ & $\mathrm{U}=2,0 ; \mathrm{NS}$ \\
Matière minérale (\% du pr. brut) & $15,5 \pm 2,9$ & $17,4 \pm 0,6$ & $\mathrm{U}=2,5 ; \mathrm{NS}$ \\
\hline Azote total $\left(\mathrm{g} \cdot \mathrm{kg}^{-1}\right.$ de poids sec) & $28,5 \pm 0,3$ & $28,2 \pm 0,9$ & $\mathrm{U}=4,0 ; \mathrm{NS}$ \\
Phosphore $\left(\mathrm{g} \cdot \mathrm{kg}^{-1}\right.$ de poids sec) & $17,1 \pm 0,4$ & $15,9 \pm 0,3$ & $\mathrm{U}=0,00 ; p \leq 0,05$ \\
Potassium $\left(\mathrm{g} \cdot \mathrm{kg}^{-1}\right.$ de poids sec) & $87,0 \pm 3,1$ & $75,2 \pm 1,5$ & $\mathrm{U}=0,00 ; p \leq 0,05$ \\
Rapport C/N & $11,0 \pm 0,2$ & $11,3 \pm 0,4$ & $\mathrm{U}=2,5 ; \mathrm{NS}$ \\
\hline
\end{tabular}

Tableau 5. Frais liés à l'exploitation des deux roselières $(€)$ et cout de revient $\left(€ \cdot \mathrm{t}^{-1}\right)-$ Costs related to the exploitation of the two reed beds (€) and cost price (€) $\left.t^{-1}\right)$.

\begin{tabular}{|c|c|c|}
\hline & $\begin{array}{l}\text { Roselière de la cabane de Moins } \\
\text { (itinéraire routier }: 10 \mathrm{~km} \text { ) } \\
\end{array}$ & $\begin{array}{l}\text { Roselière du Val de Trézence } \\
\text { (itinéraire routier : } 37 \mathrm{~km} \text { ) }\end{array}$ \\
\hline & Année 1 & Année 2 \\
\hline Frais liés à la fauche & $420 €$ & $640 €$ \\
\hline Frais liés au pressage & $604 €$ & $584 €$ \\
\hline Frais de transport & $50 € *$ & $250 €$ \\
\hline Total & $1074 €$ & $1474 € €$ \\
\hline Cout de revient & $53 € \cdot \mathrm{t}^{-1}$ & $92 € \cdot \mathrm{t}^{-1}$ \\
\hline
\end{tabular}

- un temps de fauche plus long en année 2 (5 vs 3 h) $\mathrm{du}$ fait d'un modèle de faucheuse moins adapté au roseau ;

- des frais plus élevés de déplacement du matériel ( $1 \mathrm{~h}$ aller-retour en plus $\times 2$ déplacements) ;

- des frais de transport du roseau plus élevés en année 2 .

\section{DISCUSSION}

Avec les mêmes conditions de paillage en termes de fréquence et de quantité de paille distribuée, le roseau obtient les mêmes performances vis-à-vis de la propreté des vaches et de la valeur fertilisante du compost que la paille de céréales. Il est également compétitif puisque son cout de revient varie de 53 à $92 € \cdot \mathrm{t}^{-1}$ selon la distance de la roselière à la ferme et le matériel agricole utilisé. Ceci le rend au mieux moitié moins cher, et au pire à peu près équivalent à l'achat de paille « livrée dans la cour».

\subsection{Les enseignements techniques tirés de cette étude}

Évaluée en laboratoire, la plus grande capacité qu'a montré la paille à absorber l'eau par rapport au roseau (en moyenne $+63,5 \%$ et $40,9 \%$ de gain de poids des échantillons pour les années 1 et 2, respectivement) n'a pas entrainé de performances supérieures de la litière à base de paille sur le niveau de propreté des vaches ou l'état de la litière. La mesure de la capacité d'absorption en eau de ces tiges végétales est un indicateur de leur pouvoir absorbant (Gasser, 2007 ; Lejeune, 2009) mais ne présage pas de la façon dont celles-ci vont « se comporter » en tant que litière. Les différences constatées sont certainement à mettre en lien avec la nature de ce qui est absorbé (de l'eau $v s$ de l'urine et des bouses de vaches) et des conditions de leur utilisation (immersion dans un bac d'eau vs paillage au sol). Nous pensons donc que la meilleure façon de savoir s'il peut être fait usage d'un nouveau matériau en tant que litière doit consister en 
un test en conditions réelles d'utilisation, à savoir en stabulation.

Les meilleures performances du roseau obtenues lors des essais 1 et 2 sont à nuancer du fait d'artefacts expérimentaux. En effet, nous avons observé que les vaches logées sur du roseau étaient moins sales que celles de la case "paille». Ce résultat en faveur du roseau peut s'expliquer en partie par l'utilisation lors de l'essai 1 d'une paille d'orge de mauvaise qualité, assez cassante. Le comportement atypique d'une vache dans la case "paille », qui lapait l'eau de la buvette d'abreuvement, répandant beaucoup d'eau sur une partie de la case, a également contribué au salissement accéléré de la paille.

Cette expérimentation nous a permis d'ajuster le paillage. Une fois la vache au comportement atypique retirée du lot dans l'essai 3 , et après avoir augmenté les quantités de litière distribuées au paillage, les notes de propreté des animaux se sont améliorées (sans pour autant rattraper celles du roseau). Ce résultat suggère que les quantités de litière distribuées lors des deux premiers essais n'étaient pas suffisantes pour maintenir une propreté correcte des 10-12 vaches par case. Dans nos conditions, avec trois paillages par semaine, ces quantités de litière doivent être d'environ $5 \mathrm{~kg} \cdot \mathrm{m}^{-2} \mathrm{de}$ couchage/paillage.

Un paillage plus soutenu au cours des essais 3 et 4 est sans doute à l'origine de l'augmentation constatée des températures des litières. Ceci est un phénomène bien connu des éleveurs : la température de la litière augmente avec la quantité de paille apportée au paillage. Alors que la température moyenne des litières lors des essais 1 et 2 était de 20 à $26^{\circ} \mathrm{C}$, avec très peu de différence d'une case à l'autre, celle-ci avoisinait $40{ }^{\circ} \mathrm{C}$ lors de l'essai 4. La litière du faux-roseaux était à $32^{\circ} \mathrm{C}$ et semble avoir moins fermenté que la paille ou le roseau. La température de l'aire paillée est un paramètre à surveiller eu égard au seuil critique de $40{ }^{\circ} \mathrm{C}$ à ne pas dépasser pour éviter la prolifération des germes responsables de mammites, établi en élevage bovin laitier (Ménard et al., 2004). Sachant que la mesure de la température est un indicateur utile pour optimiser les apports de litière (Roussel \& Ménard, 2004), il semble ne pas falloir dépasser lors du paillage les $5 \mathrm{~kg} \cdot \mathrm{m}^{-2} \mathrm{de}$ couchage proposés plus haut. Ramenées à une distribution quotidienne, les quantités de litière lors des essais 3 et 4 sont respectivement de 1,9 et $1,7 \mathrm{~kg} \cdot \mathrm{m}^{-2}$ paille par jour (ce sont sensiblement les mêmes quantités pour le roseau), ce qui se situe au-delà des quantités communément apportées en élevage bovin allaitant : 1 à $1,4 \mathrm{~kg} \cdot \mathrm{m}^{-2}$ paille par jour (Mille et al., 2013) contre 1 à $1,2 \mathrm{~kg} \cdot \mathrm{m}^{-2}$ paille par jour en système laitier (https://www.paysan-breton.fr/2019/11/ paillage-ni-trop-ni-trop-peu/) ou 1,1 à $1,6 \mathrm{~kg} \cdot \mathrm{m}^{-2}$ paille par jour en situation expérimentale (Ménard et al., 2004). S'écartant donc de la pratique habituelle, la recherche d'un système économe en litière dans notre cas supposerait de réduire les quantités distribuées au paillage pour revenir vers une consommation plus proche de $1,5 \mathrm{~kg} \cdot \mathrm{m}^{-2}$ paille par jour, sans que cela ne compromette à notre avis les conclusions ci-dessous de cette étude.

Nous arrivons à la conclusion que dans les mêmes conditions de paillage (fréquence et quantité de litière distribuée), le roseau donne des résultats très semblables à la paille de céréales. La surveillance de l'état sanitaire des animaux n'a révélé aucune pathologie particulière (boiterie, par exemple) dans aucune des cases. L'examen des composts issus de ces litières a montré que le roseau est monté un peu plus en température que la paille (en moyenne, $5^{\circ} \mathrm{C}$ de différence), malgré le fait que, visuellement, il ait eu tendance à moins perdre en volume par rapport à l'andain de compost de paille (A. Tricheur, observation personnelle). Vers la fin de la période de maturation des composts, la température atteignait $55-65^{\circ} \mathrm{C}$, ce qui permet une bonne hygiénisation de l'effluent. De manière générale, la valeur fertilisante des deux types de compost et leur contenu en minéraux étaient proches. Seuls le phosphore et le potassium étaient légèrement moins abondants (respectivement $7 \%$ et 13,6\%) dans le compost de roseau, comparé à celui de la paille. Les rapports $\mathrm{C} / \mathrm{N}$ sont d'environ 11 pour les deux types de compost, ce qui n'est pas très élevé (l'optimum se situant à 15-30), mais suggère, une fois épandus, un même niveau de dégradabilité au champ.

\subsection{Faisabilité du roseau comme litière et résultats économiques}

Dans les conditions de réalisation de cette étude (fréquence de paillage, quantité de litière distribuée au paillage, nombre de vaches par case, etc.), le roseau s'est révélé être une bonne alternative à la paille de céréales. Il peut être utilisé seul ou en mélange avec de la paille, même si les résultats des cases «paille + roseau » semblaient un peu moins convaincants que la paille seule (ce résultat semblant davantage dû à des artéfacts expérimentaux - problème de fuite d'une buvette lors d'un essai - qu'à la nature même des litières). Le faux-roseau peut également être utilisé mais semble plus sensible que la paille au piétinement, surtout s'il s'agit d'un lot de vaches avec des veaux (D. Durant, observation personnelle).

D'un point de vue pratique et économique, les essais de modèles de faucheuse et de presse ronde utilisées dans cette étude ont permis de préciser et actualiser les aspects techniques liés à cette pratique. L'étude a également montré que le roseau est compétitif puisque son cout de revient de $53 € \cdot \mathrm{t}^{-1}$ en année 1 est environ moitié moins cher que l'achat de paille, si on considère un cours de $100 € \cdot \mathrm{t}^{-1}$ de paille livrée (ce prix pouvant 
cependant être plus élevé en période de pénurie et de forte demande). Le cout plus élevé en année 2 $\left(92 € \cdot \mathrm{t}^{-1}\right)$ montre que la distance d'une roselière par rapport à son lieu d'utilisation est un facteur important à prendre en compte dans la « rentabilité » de l'opération (par rapport aux couts de déplacement des machines et de transport des balles). Le fait qu'un agriculteur possède le matériel pour récolter lui-même le roseau permet d'éviter les frais liés à l'entreprise. D'autre part, la fauche d'une roselière proche d'une ferme reste intéressante économiquement par rapport à la paille achetée «derrière la moissonneuse », dont nous avons estimé le cout à $60 € \cdot \mathrm{t}^{-1}$. En revanche, le caractère avantageux de cette pratique atteint probablement ses limites si on devait inclure au moins une partie des couts d'implantation d'une roselière (voire des frais liés à son entretien) dans le cout de revient $\mathrm{du}$ roseau. On peut cependant imaginer que ces couts ne soient pas pris en charge par l'agriculteur mais par des fonds publics qui encourageraient et soutiendraient financièrement l'aménagement de ces milieux favorables à la biodiversité. Il est ainsi difficile de conclure en raison du manque de références sur le sujet dans la bibliographie. Des investigations supplémentaires sont donc nécessaires afin de mieux cerner les conditions et le seuil de «rentabilité » de cette pratique.

\subsection{Implications de cette étude}

Le bilan de cette étude vient confirmer l'enthousiasme généralement constaté chez les éleveurs en Brière quant à l'utilisation du roseau en litière (Divay et al., 2018). En Charente-Maritime, la très grande majorité des exploitations de marais n'utilisent pas ce matériau et seuls quelques éleveurs continuent de pratiquer le paillage avec du roseau. Comme un peu partout en France, le courant de modernisation de l'élevage qui a démarré vers le milieu du $19^{\text {e }}$ siècle a contribué à faire disparaitre des savoir-faire acquis pendant des décennies d'utilisation raisonnée des ressources naturelles locales. Les éleveurs ont ainsi été petit à petit convaincus que des milieux tels que les roselières étaient improductifs et donc inutiles à maintenir dans le paysage, ce qui a contribué à les faire régresser. Un récent inventaire estime que la superficie totale actuelle des roselières en Charente-Maritime est de 1800 ha, dont la moitié est à enjeux forts pour l'avifaune qui les fréquente (Jomat \& Barret, 2020). Or, la présente étude a confirmé deux choses. La première est le caractère très productif d'une roselière. Nous avons relevé des biomasses de 15,7 et $14,6 \mathrm{t} \cdot \mathrm{ha}^{-1}$ pour les deux roselières, ce qui est en accord avec les niveaux de production moyens rapportés dans la littérature : $12,5 \mathrm{t} \cdot \mathrm{ha}^{-1}$ (variation de 5 à $25 \mathrm{t} \cdot \mathrm{ha}^{-1}$; LPO Alsace, 2013) et $13 \mathrm{t} \cdot \mathrm{ha}^{-1}$ (5 à $36 \mathrm{t} \cdot \mathrm{ha}^{-1}$; E.P.A., 1985). La seconde constatation porte sur l'intérêt que peut avoir le roseau pour contribuer au bon fonctionnement des exploitations en zones humides. Montrer son utilité pour les élevages pourrait être un moyen de réhabiliter (entendez ici étendre ou re-créer) ces surfaces de roselières et ce, avec un double objectif :

- un objectif environnemental, puisque les roselières rendent de nombreux services environnementaux (sites de nidification et ressources alimentaires pour des oiseaux paludicoles, participation à l'épuration de l'eau des marais, habitat typique des paysages des zones humides; Ostendorp, 1993 ; Köbbing et al., 2013);

- un objectif agricole, en valorisant économiquement ces habitats typiques des marais qui peuvent être sources de litière pour les animaux.

C'est précisément ce à quoi s'attache le concept de restauration du capital naturel (Aronson et al., 2007). Il considère qu'il y a un lien étroit entre, d'une part, la restauration des milieux dégradés, de leurs fonctionnalités et la conservation de la biodiversité qui va souvent de pair et, d'autre part, des préoccupations économiques et sociales locales (Blignaut et al., 2014). L'idée sous-jacente est que la restauration écologique bénéficie dans bien des cas à l'économie locale (Aronson et al., 2006). Ainsi, la restauration du «capital roseau » qui existait davantage autrefois sur les fermes de marais pourrait bénéficier aux éleveurs qui réaliseraient des économies sur les achats de paille et/ou percevraient des paiements pour services environnementaux (Vaissière et al., 2020). Ainsi, pailler avec du roseau pourrait-il de nouveau faire partie des pratiques des éleveurs d'aujourd'hui ? Il est peu probable qu'une ferme possède assez de roselières pour couvrir la totalité de ses besoins en litière. Pour notre cas par exemple, les besoins annuels en litière de 100 t nécessiteraient environ 12 ha de roselière (en supposant une production de $15 \mathrm{t} \cdot \mathrm{ha}^{-1}$ de la roselière et une fauche tous les deux ans). Les surfaces de roselière actuellement exploitables dans le voisinage de la ferme ne sont donc pas suffisantes au regard de notre cheptel et de celui des fermes aux alentours. Mais une surface même modeste d' 1 à 2 ha permettrait d'apporter un complément de litière et diminuer la dépendance des fermes d'élevage aux achats de paille. Il est aussi possible que certaines années difficiles, ces surfaces puissent constituer des « zones de flexibilité » en palliant la pénurie de paille et la hausse des prix liée au dérèglement climatique. Des échanges directs et de proximité entre éleveurs ayant des surfaces plus ou moins grandes de roselières au sein d'un territoire de marais pourraient ainsi se mettre en place. Il reste enfin un dernier aspect à ne pas négliger : accompagner les agriculteurs dans leurs modalités d'utilisation de ces habitats, puisqu'une mauvaise gestion pourrait 
compromette le rôle des roselières dans la fourniture des services écosystémiques. En effet, sous certaines conditions, la fauche avec exportation permet de préserver l'équilibre des roselières (Le Bihan \& Birard, 2004) en évitant les phénomènes d'atterrissement qui peuvent avoir lieu pour des roselières jamais entretenues, ce qui nécessite quelque fois un étrépage (à savoir l'élimination de la couche superficielle du sol). C'est pourquoi nous pensons que l'exportation de la matière végétale contribue à la bonne vitalité d'une roselière, en veillant cependant à ce que cette fauche ne soit pas trop fréquente (Hawke \& José, 1996). Tout l'enjeu est donc d'adopter une gestion adaptée au cycle biologique du roseau ainsi que celui des autres espèces animales qui lui sont liées (E.P.A., 1985 ; Schricke, 1986).

\section{CONCLUSIONS}

Cette étude montre que le roseau peut être utilisé en litière en élevage bovin allaitant. Cette espèce végétale pourrait retrouver une place dans les exploitations de marais, en particulier celles conduites en $A B$ qui cherchent à mieux valoriser les ressources naturelles du milieu, tout en les préservant. La tendance amorcée depuis quelques années par ce type d'agriculture, occupant des surfaces agricoles croissantes, interroge sur les nouvelles ressources à mobiliser pour que cette agriculture produise suffisamment et durablement. Le roseau pourrait ainsi être une de ces ressources, ce qui pose alors la question des modalités de restauration de ce capital naturel et les conditions de gestion des roselières, dans le respect des cycles de la nature.

\section{Remerciements}

Les auteurs remercient Pierre Roux et Michel Prieur, animaliers sur la ferme expérimentale, en tant que fervents contributeurs à cette étude.

Ils sont reconnaissants à la Fédération Départementale des Chasseurs de Charente-Maritime ainsi qu'au Département de la Charente-Maritime pour la mise à disposition des roselières. Leurs remerciements vont également à Matthieu Marquet du Parc Naturel Régional de Brière pour les avoir mis en contact avec des éleveurs utilisant le roseau comme litière, Christophe Le Bellec et Amandine Caillon (INRAE) pour leurs «coups de main» à l'expérimentation, ainsi qu'Éric Kernéîs pour la relecture de l'article.

Merci à Corentin Doublet, mais également Philippe Barre et Isabelle Litrico pour leurs conseils sur les analyses statistiques.

Cette étude a bénéficié d'une aide financière de la Région Nouvelle-Aquitaine (contrats LM-332019-20172780520 et TER20-02-2018-3455720).

\section{Bibliographie}

Altieri M.A., 1996. Agroecology: the science of sustainable agriculture. $2^{\text {nd }}$ ed. Boca Raton, FL, USA: CRC Press.

Aronson J., Clewell A.F., Blignaut J.N. \& Milton S.J., 2006. Ecological restoration: a new frontier for nature conservation and economics. J. Nat. Conserv., 14, 135139, doi: 10.1016/j.jnc.2006.05.005

Aronson J., Milton S.J. \& Blignaut J.N., 2007. Restoring natural capital: science, business and practice. Washington: Island Press.

Bastien D., Cartier P. \& Lucbert J., 2006. Grille de notation de la propreté des bovins vivants. Compte-rendu 170632 005. Paris : Institut de l'Élevage.

Berthelot P.-J. et al., 2003. L'utilisation des plaquettes de bois en litière animale. Étude de pratiques en BasseNormandie. ENSAR-AILE.

Blignaut J., Aronson J. \& de Groot R., 2014. Restoration of natural capital: a key strategy on the path to sustainability. Ecol.Eng., 65,54-61, doi: 10.1016/j.ecoleng.2013.09.003

Capdeville J. \& Ménard J.-L., 2003. Possibilités de substitution de la paille par d'autres matériaux de litière. Dossier sécheresse 2003. Paris : Institut de l'Élevage.

Divay Q., Hadj-Abed S., Martin A. \& Thévenard G., 2018. Roselières de Brière. Rapport de Master 2 - Écologie et éco-ingénierie des zones humides. Angers, France: Université d'Angers.

Durant D. \& Kernéis E., 2010. Contribution of a system experiment in designing a mixed crop-livestock farming system aimed at i) improving self-sufficiency, and ii) producing biodiversity and benefiting from it. In: Cirad, Inra, SupAgro, eds. Proceedings of the ISDA conference, Innovation and sustainable development in agriculture and food, 28 June-1 July, 2010, Montpellier, France.

Durant D. et al., 2020. Comment évoluer vers davantage d'autonomie au sein des systèmes de polycultureélevage ?: l'expérience d'une ferme expérimentale en marais. Fourrages, 241, 21-34.

E.P.A., 1985. Le roseau. In: Établissement Public d'Aménagement / Office National de la Chasse, eds. Les hélophytes - récolte et valorisation des végétaux herbacés en zone humide. Paris: Ministère de l'Agriculture, 20189.

Gasser M.-O., 2007. Capacité d'absorption en eau des litières. Rapport final. Québec, Canada : IRDA.

Gliesmann S., 1998. Agroecology: ecological processes in sustainable agriculture. $3^{\mathrm{d}}$ ed. Boca Raton, FL, USA: CRC Press.

Goergen P., 1996. Entre patrimoine naturel et patrimoine architectural: le chaume de marais, approche ethnologique d'un parcours. Rapport final PNR de Brière, de Brotonne, du Cotentin et du Bessin. Paris : Ministère de la Culture.

Hawke C.J. \& José P.V., 1996. Reedbed management. For commercial and wildlife interests. Sandy, UK: Royal Society for the Protection of Birds. 
Jomat L. \& Barret V., 2020. Études sur les roselières de Charente-Maritime - Utilisation de l'avifaune comme indicateur de la qualité des roselières. Rapport intermédiaire. Rochefort, France: Ligue pour la Protection des Oiseaux.

Köbbing J.F., Thevs N. \& Zerbe S., 2013. The utilization of reed (Phragmites australis): a review. Mires Peat, 13, $1-14$.

Le Barz C., Michas M. \& Fouque C., 2009. Les roselières en France métropolitaine: premier inventaire (19982008). Faune Sauvage, 283, 14-26.

Le Bihan J. \& Birard C., 2004. Recueil d'expériences en matière de gestion de roselières. Paris : Fédération des parcs naturels régionaux de France.

Lejeune J.-P., 2009. Étude comparative d'une litière pour chevaux à base de chanvre. Vielsalm, Belgique : Centre européen du Cheval.

LPO Alsace, 2013. Roselières, prairies et cultures à but énergétique et biodiversité : étude des opportunités et des risques pour la nature. Rapport d'étude. Rosenwiller, France : Ligue pour la Protection des Oiseaux.

Madelrieux S. et al., 2017. Autonomie territoriale pour l'approvisionnement des exploitations: outils d'évaluation et application au territoire du Sud-Isère. In: Actes de la Conférence, Les polycultures-élevages, valoriser les atouts pour la transition écologique, 10-11 Octobre 2017, AgroSup Dijon, Dijon, France.

Malterre M.-F., 2019. Litière - Ils trouvent des alternatives à la paille. France Agricole, 3818, 48-53.

Ménard J.-L. et al., 2004. Contamination bactérienne d'une litière de stabulation libre paillée : effet de la fréquence de paillage et proposition d'une méthode pour son évaluation. Renc. Rech. Ruminants, 11, 333-336.

Mille S., Capdeville J.\& Blanchin J.-Y., 2013. Les bâtiments bovins de demain : économes en paille et producteurs d'énergie. Communication orale au Sommet de l'Élevage 2013, http://idele.fr/fileadmin/medias/Documents/ Batiments_bovin_de_demain.pdf, (1/10/2021).
Ostendorp W., 1993. Reed bed characteristics and significance of reeds in landscape ecology. Limnol. Aktuell, 5, 149-160.

Roussel P. \& Ménard J.-L., 2004. Température des litières : démarche de conseil technique en élevage. Angers, France: Institut de l'Élevage, Chambre Régionale d'Agriculture des Pays de la Loire, https://pays-de-laloire.chambres-agriculture.fr/fileadmin/user_upload/ National/FAL_commun/publications/Pays_de_la_ Loire/2009_et_ant/plaquette_04_temperature_des_ litieres.pdf, (1/10/2021).

Rousset N. et al., 2014. Les litières en élevage : identification, test et évaluation des techniques ou des pratiques consistant à mieux gérer les litières avec moins de matériaux. Innov. Agron., 34, 403-415.

Schricke V., 1986. Le roseau : biologie et écologie. Bull. Mensuel Off. Natl Chasse, 105, 23-35.

Schricke V., 1988. Le roseau, les autres hélophytes. Techniques de culture et de récolte. Utilisation et valorisation économique. Bull. Mensuel Off. Natl Chasse, 120, 28-36.

Vaissière A.-C. et al., 2020. Biodiversity offsets and payments for environmental services: clarifying the family ties. Ecol. Econ., 169, 1-10, doi:10.1016/j. ecolecon.2019.106428

Van Soest P.J., Robertson J.B. \& Lewis B.A., 1991. Methods for dietary fiber, neutral detergent fiber, and nonstarch polysaccharides in relation to animal nutrition. J. Dairy Sci., 74, 3583-3597, doi: 10.3168/jds.S00220302(91)78551-2

Van Weyenberg S. et al., 2015. Feasibility of Miscanthus as alternative bedding for dairy cows. Vet. Med., 60(3), 121-132, doi: 10.17221/8058-VETMED 